\title{
Neurological involvement in Kawasaki disease: a retrospective study
}

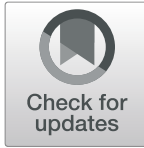

Xiaoliang Liu ${ }^{1,2,3}$, Kaiyu Zhou ${ }^{1,2,3,4}$, Yimin Hua ${ }^{1,2,3,4}$, Mei Wu ${ }^{1,5}$, Lei Liu ${ }^{1,5}$, Shuran Shao ${ }^{1,5}$ and Chuan Wang ${ }^{1,2,3,4^{*}}$ (D)

\begin{abstract}
Background: Kawasaki disease (KD) is an acute, self-limiting systemic vasculitis that predominately affects children. Neurological involvement is a known complication of KD, however, its association with KD severity remains elusive. We aimed to systematically describe the general manifestations of neurological involvement in KD, determine whether neurological involvement is a marker of disease severity in patients with $\mathrm{KD}$, and assess the relationship of such involvement with intravenous immunoglobulin (IVIG) resistance and coronary artery lesions (CALs).

Methods: We retrospectively reviewed data from 1582 patients with KD between January 2013 and December 2017. Profiles of patients with neurological symptoms (group $A, n=80$ ) were compared to those of gender- and admission date-matched patients without neurological involvement (group $B, n=512$ ). Multivariate logistic regression analyses were performed to determine whether neurological involvement was significantly associated with IVIG resistance.
\end{abstract}

Results: Neurological involvement was observed in 5.1\% (80/1582) of patients with KD. The neurological manifestations were diffuse, presenting as headache (13/80, 16.3\%), convulsions (14/80, 17.5\%), somnolence (40/80, $50.1 \%)$, extreme irritability $(21 / 80,26.3 \%)$, signs of meningeal irritation (15/80, 18.8\%), bulging fontanelles (7/80, $8.8 \%)$, and facial palsy (1/80,1.3\%). Neurological symptoms represented the initial and/or predominant manifestation in $47.5 \%$ (38/80) of patients with KD. The incidence of IVIG resistance and levels of inflammatory markers were higher in group A than in group B. However, neurological involvement was not an independent risk factor for IVIG resistance or CALS.

Conclusion: Rates of neurological involvement were relatively low in patients with KD. Neurological involvement was associated with an increased risk of IVIG resistance and severe inflammatory burden. Our results highlight the need for pediatricians to recognize KD with neurological involvement and the importance of standard IVIG therapy.

Trial registration: Retrospectively registered.

Keywords: Neurological involvement, Kawasaki disease, Intravenous immunoglobulin resistance, Coronary artery lesions, Vasculitis

\footnotetext{
* Correspondence: 805101396@qq.com

'Department of Pediatric Cardiology, West China Second University Hospital,

Sichuan University, No. 20, 3rd section, South Renmin Road, Chengdu

610041, Sichuan, China

${ }^{2}$ Key Laboratory of Birth Defects and Related Diseases of Women and

Children (Sichuan University), Ministry of Education, Chengdu, Sichuan, China

Full list of author information is available at the end of the article
}

(C) The Author(s). 2020 Open Access This article is licensed under a Creative Commons Attribution 4.0 International License, which permits use, sharing, adaptation, distribution and reproduction in any medium or format, as long as you give appropriate credit to the original author(s) and the source, provide a link to the Creative Commons licence, and indicate if changes were made. The images or other third party material in this article are included in the article's Creative Commons licence, unless indicated otherwise in a credit line to the material. If material is not included in the article's Creative Commons licence and your intended use is not permitted by statutory regulation or exceeds the permitted use, you will need to obtain permission directly from the copyright holder. To view a copy of this licence, visit http://creativecommons.org/licenses/by/4.0/ The Creative Commons Public Domain Dedication waiver (http://creativecommons.org/publicdomain/zero/1.0/) applies to the data made available in this article, unless otherwise stated in a credit line to the data. 


\section{Background}

Kawasaki disease (KD) is an acute, self-limiting form of systemic vasculitis that predominately affects children between the ages of 6 months and 5 years. Clinically, KD is typically characterized by persistent fever, bilateral non-exudative conjunctivitis, erythema of the lips and oral mucosa, changes in the extremities, rash, and cervical lymphadenopathy [1]. In addition, KD can affect multiple organs and tissues including the pulmonary and gastrointestinal systems, thereby increasing the risk of coronary artery lesions (CALs) associated with delayed diagnosis and/or intravenous immunoglobulin (IVIG) resistance [2-4].

Although neurological involvement has been identified as a complication of $\mathrm{KD}$, with an incidence of 1 to $30 \%$ $[5,6]$, its clinical features are diverse, and there are few reported cases in the literature [7-18]. Thus, pediatricians may be unfamiliar with the characteristics, course, clinical manifestations, diagnosis, treatment, and prognosis of KD with neurological involvement. Furthermore, the familiar diagnostic features of KD often appear sequentially and become obvious only after the initial neurological presentation, possibly resulting in unnecessary/inadequate treatment and/or therapeutic delay, and in turn increasing the risk of developing IVIG resistance and CALs [19]. The associations between neurological involvement and IVIG resistance and CALs are, however, not well clarified. Therefore, in the present study, we aimed to systematically describe the general manifestations of neurological involvement in $\mathrm{KD}$, determine whether neurological involvement is a marker of disease severity in patients with $\mathrm{KD}$, and assess the relationship of such involvement with IVIG resistance and CALs.

\section{Methods}

We retrospectively reviewed data for a total of 1582 patients diagnosed with KD between January 2013 and December 2017 at the West China Second University Hospital of Sichuan University (WCSUH-SCU). Written informed consent was obtained from the parents following a full explanation of the nature of the study. The University Ethics Committee on Human Subjects at Sichuan University approved the study.

Neurological involvements were recorded, including somnolence, extreme irritability, headache, convulsions, signs of meningeal irritation, bulging fontanelles, hemiplegia, facial nerve palsy, sensorineural hearing loss, and aseptic meningitis (pleocytosis in the cerebrospinal fluid $\left.(\mathrm{CSF}) \geq 15 \times 10^{6} / \mathrm{L}\right)$. The presences of somnolence and extreme irritability were documented in accordance with standard definitions of Richmond Agitation Sedation Scale (RASS) [20, 21] when reported by caregivers and/ or directly observed during the acute stage of hospitalization. Headache was defined based on clinical history and physical examination using an ageappropriate pain assessment scale (i.e., the Face, Legs, Activity, Cry, Consolability Scale (FLACC Scale) [22], Wong-Baker Faces Pain Rating Scale [23]). Abnormal CSF results were defined as follows: pleocytosis $\geq 15 \times$ $10^{6} / \mathrm{L}$, protein level $>430 \mathrm{mg} / \mathrm{L}$, glucose $<2.8 \mathrm{mmol} / \mathrm{L}$, chloride $<120 \mathrm{mmol} / \mathrm{L}$. The convulsions, signs of meningeal irritation, bulging fontanelles, aseptic meningitis, and facial nerve palsy, were identified by clinical history, CSF results, physical and neuroimaging examinations. Congenital, trauma-related, or infectious causes for aforementioned neurological symptoms were excluded by negative family history or prior history of febrile convulsion and epilepsy, cranial computed tomography (CT)/magnetic resonance imaging (MRI), electroencephalogram, lumbar puncture, biochemical, and metabolic investigations. Furthermore, all these neurological symptoms and/or signs regressed or disappeared after IVIG treatment.

Diagnoses of KD were made by two pediatricians (including at least one KD specialist) in accordance with the 2004 American Heart Association Recommendations for KD [20]. Kawasaki disease shock syndrome (KDSS) was defined as patients with KD presenting systolic hypotension for age, a sustained decrease in systolic blood pressure from baseline of $\geq 20 \%$, or clinical signs of poor perfusion [24]. In order to examine whether neurological involvement contributes to the risk of IVIG resistance and CALs among patients with $K D$, the profiles of patients who exhibited any neurological symptoms (group A) were compared to those of gender- and admission date-matched patients without neurological involvement (group B). All available clinical details, laboratory examination parameters, echocardiogram data, treatment results, and follow-up data were systematically collected and analyzed.

In accordance with 2004 American Heart Association Recommendations, the standard IVIG therapy refers to a dose of $2 \mathrm{~g} / \mathrm{kg}$ given as a single intravenous infusion after the diagnosis of KD established. Other usage of IVIG such as $1 \mathrm{~g} / \mathrm{kg}$ for 2 days or $500 \mathrm{mg} / \mathrm{kg}$ for 4 days were defined as non-standard IVIG therapy. High dose of aspirin (30-50 mg/kg/day) was also given orally. After the patient's fever had resolved, the dose of aspirin was decreased to $3-5 \mathrm{mg} / \mathrm{kg} /$ day and continued for $6-8$ weeks. In patients with CALs, aspirin treatment continued until the patient exhibited no signs of coronary changes. Anticoagulants and antiplatelet therapy were also performed in accordance with 2004 American Heart Association Recommendations. Patients with recurrent or persistent fever for $\geq 36 \mathrm{~h}$ after the first IVIG administration were treated with a second course of IVIG $(2 \mathrm{~g} / \mathrm{kg})$. Furthermore, high-dose pulsed intravenous methylprednisolone 
(20-30 mg/kg) treatment was administered for 3 days in patients with recurrent or persistent fever even after an additional IVIG. IVIG resistance was defined as persistent or recurrent fever (oral temperature $\geq 38.0^{\circ} \mathrm{C}$ ) or other clinical signs of KD at least $36 \mathrm{~h}$ but not longer than 7 days after the first IVIG infusion [25].

CALs were classified based on normalized Z scores for body surface area (BSA; units of standard deviation from the mean, normalized for BSA), as follows: no involvement ( $Z$ score $<2.0$ ), dilation $(Z$ score $\geq 2.0$ to $<2.5$ ), aneurysm $\quad(Z \quad$ score $\geq 2.5 ; \quad Z \quad$ score $\geq 10$ for giant aneurysm). We evaluated the maximal internal diameters of the right coronary artery (RCA), left anterior descending artery (LAD), and left circumflex coronary artery (LCX) [1]. In accordance with our institutional protocol, patients underwent standardized echocardiography by two pediatric ultrasonologists during the acute phase and 6 to 8 weeks later during follow-up evaluations in the cardiology clinic, until CALs had resolved.

\section{Statistical analysis}

All data were analyzed using SPSS version 21.0 (SPSS Inc. Chicago, IL, USA). Quantitative data are presented as the mean and range or mean \pm standard deviation (SD), while qualitative data are expressed as n/\%. Shapiro-Wilk and homogeneity of variance tests were used to confirm that quantitative data from different groups exhibited a normal distribution with homogeneity of variance. Differences in quantitative data between group $A$ and group B were assessed using independent Samples t-tests or Mann-Whitney U-tests. To assess nonparametric dichotomous and non-dichotomous variables, we used contingency tables/Fisher's exact tests and regression analyses with dummy variables, respectively. Multivariate logistic regression analyses were performed to determine whether neurological involvement was significantly associated with IVIG resistance. These analyses included variables that significantly differed between patients with and without IVIG resistance. The level of statistical significance was set at $P<0.05$ (two-tailed).

\section{Results}

Neurological involvement was observed in 80 patients with KD (46 males, 34 females; M:F ratio of 1.35:1). Age in patients with neurological involvement ranged from 1 to 115 months. Sixty-percent $(48 / 80)$ of patients were admitted via the Cardiology Department, 20.0\% (16/80) via the Neurology Department, $16.2 \%(13 / 80)$ via the Pediatric Intensive Care Unit (ICU), 2.5\% (2/80) via the Infectious Disease Department, and 1.3\% (1/80) via other department. Neurological symptoms included headache (13/80, 16.3\%), convulsions (14/80, 17.5\%), somnolence (40/80, 50.1\%), extreme irritability $(21 / 80,26.3 \%)$, signs of meningeal irritation (15/80, 18.8\%), bulging fontanelles $(7 / 80,8.8 \%)$, and facial nerve palsy $(1 / 80$, $1.3 \%)$. The presence of hemiplegia, and sensorineural hearing loss were not observed in our cohort. Neurological involvement was present in $41.3 \%$ (33/80) of patients prior to the occurrence of familiar diagnostic features of KD, while $28.8 \%$ (23/80) presented after. Neurological symptoms represented the initial and/or predominant manifestation in $47.5 \%(38 / 80)$ of patients. Thirty-seven patients with neurological involvement underwent CSF examination, 12 of whom were considered to have aseptic meningitis $(12 / 80,15.0 \%)$ based on nucleated cell counts $>15 \times 10^{6} / \mathrm{L}$ (mean: $110 \times 10^{6} / \mathrm{L}$; range: 17 to $\left.340 \times 10^{6} / \mathrm{L}\right)$. Among these 12 patients, $66.7 \%$ (8/12) exhibited lymphocyte predominance (mean: $53.9 \%$, range: $6.0 \sim 100.0 \%$ ), while the remaining $33.3 \%$ (4/12) exhibited neutrophil predominance (mean: $33.8 \%$, range: $0.0 \sim 93 \%$ ). The remaining 25 patients had normal CSF results. Protein, glucose, chloride, and lactate dehydrogenase $(\mathrm{LDH})$ levels in the CSF were within the normal range. The results of bacteriologic and viral investigations in the CSF were negative in all patients. Biochemical and metabolic investigations (e.g., blood glucose, electrolytes, ammonia, amino acid chromatography, organic acids) yielded normal results. Almost all KD patient exhibiting neurological involvement had received cranial CT examinations but no positive findings were noted. Meningeal enhancement was also not observed in any patients with aseptic meningitis. Meanwhile, 14 patients suffering from convulsions had further underwent MRI, but no abnormalities were found. In patients with CSF examination, the proportion of males was significantly higher among patients with KD with abnormal results of CSF than without; clinical characteristics and laboratory profiles showed no differences, as summarized in supplemental material 1.

A detailed comparison of clinical data between groups $\mathrm{A}$ and $\mathrm{B}$ is presented in Table 1 . There were noted no significant differences in the gender ratio, average age, fever duration on admission/before initial IVIG, incidence of incomplete KD or typical clinical features, and the rate of delayed IVIG treatment between the groups (all $P>0.05$ ). The occurrence of KDSS was significantly higher in patients from group $A$ than that those from group B $(12.5 \%$ vs $0.2 \%, P<0.001)$. The duration of antibiotic treatment was significantly longer in group A than in group $\mathrm{B}(6.4 \pm 2.5$ vs. $4.8 \pm 2.2$ days, $\mathrm{P}<0.001)$. In addition, rates of IVIG resistance $(27.5 \%$ vs. $13.7 \%, P=$ $0.003)$ and intravenous steroid treatment $(16.3 \%$ vs. $7.4 \%, P=0.016$ ) were higher in group A than in group $\mathrm{B}$. Similar results were obtained when comparing patients in the two subgroups admitted via Non-Cardiology departments $(16.3 \%$ vs. $1.6 \%, \mathrm{P}<0.001)$. Prior to the initial IVIG treatment, the following were significantly higher in group A than in group B: white blood cell (WBC), 
Table 1 The comparison of demographic, clinical characteristic and laboratory results of Kawasaki disease between the two groups

\begin{tabular}{|c|c|c|c|}
\hline & Group $A^{a}$ & Group B ${ }^{b}$ & $P$ value \\
\hline \multicolumn{4}{|l|}{ Clinical characteristic } \\
\hline Patients, n & 80 & 512 & - \\
\hline Sex, male, n(\%) & $46(57.5)$ & 296(57.8) & 0.526 \\
\hline Age, months & $33.0 \pm 26.8$ & $30.4 \pm 23.4$ & 0.437 \\
\hline Fever duration on admission, days & $5.5 \pm 3.0$ & $5.5 \pm 2.8$ & 0.893 \\
\hline Incomplete Kawasaki disease, n(\%) & $34(42.5)$ & 192(37.5) & 0.390 \\
\hline Kawasaki disease shock syndrome, n(\%) & 10(12.5) & $1(0.2)$ & $<0.001^{*}$ \\
\hline \multicolumn{4}{|l|}{ Typical clinical manifestations } \\
\hline Fever, n(\%) & $80(100.0)$ & $512(100.0)$ & - \\
\hline Rash, n(\%) & $60(75.0)$ & $395(77.1)$ & 0.670 \\
\hline Edema \& erythema of the extremities, $n(\%)$ & $46(57.5)$ & 297(58.0) & 0.863 \\
\hline Bilateral bulbar conjunctival injection, n(\%) & $70(87.5)$ & 464(90.6) & 0.480 \\
\hline Erythema of oral and pharyngeal mucosa, n(\%) & $72(90.0)$ & 470(91.8) & 0.524 \\
\hline Cervical lymphadenopathy, n(\%) & $38(47.5)$ & $220(43.1)$ & 0.470 \\
\hline \multicolumn{4}{|l|}{ Treatment } \\
\hline The duration of antibiotic, days & $6.4 \pm 2.5$ & $4.8 \pm 2.2$ & $<0.001^{*}$ \\
\hline Fever duration before IVIG, days & $6.4 \pm 4.0$ & $6.3 \pm 3.0$ & 0.782 \\
\hline The delayed treatment of initial IVIG (>10 days) & $4(5.0)$ & $21(4.1)$ & 0.762 \\
\hline Failure to respond to initial IVIG therapy, n(\%) & $22(27.5)$ & 70(13.7) & $0.003^{*}$ \\
\hline Patients from Cardiology Department, n(\%) & $9(11.3)$ & $62(12.1)$ & 0.837 \\
\hline Patients from non-Cardiology Department, n(\%) & $13(16.3)$ & $8(1.6)$ & $<0.001^{*}$ \\
\hline Intravenous and/or Oral steroid treatment, n(\%) & 13(16.3) & $38(7.4)$ & $0.016^{*}$ \\
\hline \multicolumn{4}{|l|}{ Cardiac complications } \\
\hline Coronary artery lesions, n(\%) & $12(15.0)$ & $50(9.8)$ & 0.220 \\
\hline Dilatation, n(\%) & $11(13.8)$ & $45(8.8)$ & 0.180 \\
\hline Aneurysms, n(\%) & $1(1.3)$ & $5(1.0)$ & 0.821 \\
\hline Valve regurgitation, n(\%) & $8(10.0)$ & $32(6.3)$ & 0.229 \\
\hline Cardiac enlargement, n(\%) & 10(12.5) & $37(7.2)$ & 0.118 \\
\hline Pericardial effusion, n(\%) & $4(5.0)$ & $12(2.3)$ & 0.253 \\
\hline \multicolumn{4}{|l|}{ Blood examination features } \\
\hline White blood cell count, $\times 10^{9} / \mathrm{L}$ & $15.5 \pm 5.8$ & $14.3 \pm 5.4$ & $0.042^{*}$ \\
\hline Neutrophil rate, \% & $70.3 \pm 17.0$ & $66.1 \pm 14.7$ & $0.014^{*}$ \\
\hline Lymphocyte rate, \% & $19.6 \pm 12.3$ & $25.1 \pm 12.9$ & $<0.001^{*}$ \\
\hline C-reactive proteins, mg/L & $96.0 \pm 53.1$ & $79.3 \pm 47.8$ & $0.003^{*}$ \\
\hline Erythrocyte sediment rate, $\mathrm{mm} / \mathrm{h}$ & $69.4 \pm 34.1$ & $65.1 \pm 29.1$ & 0.326 \\
\hline Platelet, $\times 10^{9} / \mathrm{L}$ & $325.0 \pm 117.0$ & $343.9 \pm 115.0$ & 0.164 \\
\hline Hemoglobin, g/L & $104.0 \pm 13.3$ & $108.4 \pm 10.9$ & $0.001^{*}$ \\
\hline Alanine transaminase, $U / L$ & $75.2 \pm 83.9$ & $67.8 \pm 84.4$ & 0.516 \\
\hline Aspartate transaminase, U/L & $58.9 \pm 88.3$ & $49.5 \pm 57.0$ & 0.233 \\
\hline Albumin, g/L & $34.4 \pm 7.0$ & $37.7 \pm 5.0$ & $<0.001^{*}$ \\
\hline Total bilirubin, umol/L & $10.3 \pm 15.3$ & $7.7 \pm 7.9$ & $0.026^{*}$ \\
\hline $\mathrm{Cr}, \mathrm{umol} / \mathrm{L}$ & $33.5 \pm 14.6$ & $28.2 \pm 13.8$ & $0.003^{*}$ \\
\hline Serum sodium, mmol/L & $135.3 \pm 4.8$ & $136.3 \pm 7.1$ & 0.098 \\
\hline
\end{tabular}

The data are presented as mean \pm standard deviation (SD) for quantitative variables and as $\mathrm{n} / \%$ for qualitative data as appropriate. ${ }^{*} P<0.05$

IVIG Intravenous immunoglobulin

${ }^{a}$ Group A, KD patients had neurological involvement

${ }^{\mathrm{b}}$ Group B, KD patients had no neurological involvement 
neutrophil percentages, $\mathrm{C}$-reactive protein (CRP), total bilirubin (TB), and creatinine $(\mathrm{Cr})$ (all $P<0.005)$. However, hemoglobin $(\mathrm{Hb})$ levels, albumin (ALB) levels, and lymphocyte percentage were lower in group $A$ than in group $\mathrm{B}($ all $\mathrm{P}<0.005)$. Rates of cardiac complications including CALs, valve regurgitation, cardiac enlargement, and pericardial effusion were relatively higher in group A than in group B. These differences were not statistically significant. The $z$ scores of CALs in patients from group A also did not significantly differ from that in patients from group B $(1.59 \pm 0.40$ vs $1.57 \pm 0.37, P=$ 0.709). In comparison with patients of group $B$, somnolence as one subtype presentation in KD with neurological involvement showed a higher incidence of IVIG resistance but similar incidence of CALs, no significant difference of IVIG resistance and CALs was observed in patients for other subtype presentations (Table 2). Additionally, it found that neurological involvement was not an independent risk factor for IVIG resistance in patients with KD (Supplementary material 2 and 3).

\section{Discussion}

To our knowledge, the present study is the first to systemically analyze neurological involvement among patients with KD, and whether such involvement is associated with an increased risk of IVIG resistance and CALs. Our findings indicate that the incidence of neurological involvement was relatively low among patients with $\mathrm{KD}(5.1 \%)$. In many of these patients, neurological involvement was associated with diffuse clinical symptoms and accounted for the initial and/or predominant presentation. Although we observed no significant delays in diagnosis or IVIG treatment between those with and without neurological involvement, patients with neurological involvement (particularly those admitted via noncardiology departments) exhibited a higher incidence of non-standard IVIG therapy and IVIG resistance. Inconsistent with our hypothesis, we observed no significant differences in the rate of CALs occurrence between patients with and without neurological involvement.

The neurological manifestations of KD were diffuse, presenting as headache, convulsions, somnolence, extreme irritability, signs of meningeal irritation, bulging fontanelles, and facial nerve palsy. The presence of hemiplegia and sensorineural hearing loss were not found in our cohort. Fortunately, almost all above neurological symptoms regressed or disappeared after IVIG treatment and the overall prognosis of patients with KD exhibiting neurological involvement was satisfactory. Although the incidence of incomplete KD was higher than expected (42.5\%) in patients with neurological involvement, IVIG treatment was delayed in only $5.0 \%$ of patients. Our findings indicated that levels of inflammatory markers (WBC count, neutrophil percentage, CRP levels, etc.) were significantly increased in patients with neurological involvement. In addition, $15.0 \%$ of patients with neurological involvement undergoing CSF examination were diagnosed with aseptic meningitis based on increases in nucleated cell counts without alterations in other CSF indices. A total of $66.7 \%$ exhibited lymphocyte predominance, while the remaining patients exhibited neutrophil predominance. In patients undergoing CSF examination, those with aseptic meningitis only were more likely females, whereas their typical clinical manifestations of $\mathrm{KD}$, laboratory results, treatment, and prognosis did not differ from those without. The prevalence of delayed treatment in patients with neurological involvement was similar to patients without neurological involvement, but a second round of IVIG and/or steroid treatment were more often observed in patients with neurological involvement. However, in subtype neurological symptoms, patients only presenting somnolence were more likely to develop a higher incidence of IVIG resistance and patients with other neurological symptoms showed no significant difference, inconsistent with prior studies [5, 14]. In addition, almost all KD patient exhibiting neurological involvement had received cranial CT examinations but no positive findings were noted. Meningeal enhancement was also not observed in any patients with aseptic meningitis. Meanwhile, 14 patients suffering from convulsions had further underwent MRI, but no abnormalities were found. However, despite the rarity, some severe and fatal cerebral vascular complications such as artery aneurysms and/or arterial stroke had been reported in KD patients [26]. Therefore, clinicians should also alert the possibility of aforementioned severe

Table 2 Comparison of IVIG resistance and CALS of KD between patients with and without neurological involvement

\begin{tabular}{|c|c|c|c|c|c|c|c|c|}
\hline & \multicolumn{7}{|c|}{ Neurological involvement $(n=80)$} & \multirow{2}{*}{$\begin{array}{l}\text { Group B } \\
(n=512)\end{array}$} \\
\hline & $\begin{array}{l}\text { Somnolence } \\
(n=40,50.1 \%)\end{array}$ & $\begin{array}{l}\text { Convulsion } \\
(n=14,17.5 \%)\end{array}$ & $\begin{array}{l}\text { Headache } \\
(n=13,16.3 \%)\end{array}$ & $\begin{array}{l}\text { Extreme irritability } \\
(n=21,26.3 \%)\end{array}$ & $\begin{array}{l}\text { Positive sign of } \\
\text { meningeal irritation } \\
(n=15,18.8 \%)\end{array}$ & $\begin{array}{l}\text { Bulgin fontanel } \\
(n=7,8.8 \%)\end{array}$ & $\begin{array}{l}\text { Facial nerve } \\
\text { palsy }(n=1,1.3 \%)\end{array}$ & \\
\hline IVIG resistance, n(\%) & $14(35.0)^{a}$ & $3(21.4)^{b}$ & $4(30.8)^{b}$ & $6(28.6)^{b}$ & $4(26.7)^{b}$ & $0(0.00)^{b}$ & $0(0.00)^{b}$ & 70(13.9) \\
\hline CALs, n(\%) & $4(10.0)^{b}$ & $2(14.3)^{b}$ & $0(0.00)^{b}$ & $3(14.3)^{b}$ & $4(26.7)^{b}$ & $1(14.3)^{b}$ & $0(0.00)^{b}$ & 50(9.6) \\
\hline
\end{tabular}


neurological complications, particularly in KD patients with prolonged fever or severe progression, and some specific neuroimaging evaluation such as magnetic resonance angiography (MRA), angiography and even single-photon emission computed tomography (SPECT), should be performed if it is necessary.

As expected, our data indicated that KD patients with neurological involvement appear to have a higher incidence of IVIG resistance. Despite the underlying mechanisms not entirely clear, several explanations could be hypothesized. Firstly, consistent with the study conducted by Korematsu et al. [27], increased levels of inflammatory markers and nucleated cell counts in the CSF were observed in KD patients with neurological involvement. Indeed, increase in the inflammatory burden has been found to be associated with IVIG resistance. Secondly, despite the incidence of delay in IVIG administration being relatively lower, a high incidence of nonstandard IVIG therapy was observed, particularly in patients admitted via the non-Cardiology departments. It was noteworthy that $100 \%$ patients with neurological involvement admitted via the Neurology Department received non-standard IVIG treatment ( $1 \mathrm{~g} / \mathrm{kg}$ for 2 days) due to suspected immune disease, while more than 50\% of patients admitted via the Pediatric ICU and Infectious Disease Department received IVIG treatment at a dose of $500 \mathrm{mg} / \mathrm{kg}$ for an initial consideration of severe infectious disease. Substantial evidence has suggested that non-standard treatment could predispose $\mathrm{KD}$ patients to a higher risk of IVIG resistance [28-31]. However, a further multivariate logistic regression analysis failed to demonstrate neurological involvement as an independent risk factor for IVIG resistance in patients with KD. This controversial result may be explained by a correlation between serum inflammatory markers and the presence of neurological involvement. Alternatively, neurological involvement may be less sensitive as a biomarker for IVIG resistance prediction compared to serum inflammatory markers such as WBC count, neutrophil percentages, CRP level, TB level, and Cr level.

Another important finding in our study was the negative associations of neurological involvement including the subtype of neurological presentations and CALs, which appears to be inconsistent with previous studies $[5,14]$. However, almost all these studies represented sporadic cases of KD with neurological involvement and did not provide statistical support for the relationship between CALs and neurological involvement [7-10]. Stowe reported that the rate of CALs was more than twice as high in patients with KD presenting with facial nerve palsy than in those with untreated KD [14]; however, the study might have been affected by selection bias. In contrast, patients with KD presented diverse neurological manifestations in the present study, more accurately reflecting the outcome of $\mathrm{KD}$ with neurological involvement. It was demonstrated that IVIG treatment could reduce the occurrence of coronary artery abnormalities in the acute phase of KD [32]. Even non-standard IVIG treatment $(500 \mathrm{mg} / \mathrm{kg}$ for 4 days, or $1 \mathrm{~g} / \mathrm{kg}$ for 2 days) also tends to reduce the incidence of new coronary artery abnormalities [28]. Therefore, the negative associations between neurological involvement and CALs in KD patients in this study could be partly explained by the timely IVIG treatment despite a high incidence of non-standard IVIG therapy observed.

This study possesses some limitations of note, including its retrospective and cross-sectional design. In addition, given the prevalence of neurological involvement in our patients, our study might have been affected by selection bias. Indeed, as our institution is the largest pediatric medical center in Southwest China, we encounter a greater proportion of complex KD cases than other institutions. Lastly, the definitions of some neurological symptoms including somnolence, extreme irritability and headache were to be with a certain degree of subjectivity, which may not be completely avoided in the present study. However, lots of efforts had been made to avoid this limitation. Several scales and/or tools were applied to reduce the possibilities of subjective evaluations. Moreover, the proportion of patients with KD exhibiting neurological involvement affected by subjective judgement was relatively small, and it may not affect our main findings and the conclusions of our study.

\section{Conclusion}

Our findings indicate that the incidence of neurological involvement is relatively low among patients with $\mathrm{KD}$. Although no significant delays in diagnosis/IVIG treatment were observed, patients with neurological involvement were more likely to develop IVIG resistance and exhibit more severe inflammatory burden than patients without neurological involvement. The overall prognosis of patients with KD exhibiting neurological involvement was satisfactory, as we observed no associations between neurological involvement and the development of CALs. Owing to atypical clinical profile of some KD patients in early disease stage and limited knowledge of clinicians on the neurological involvements in KD, the usage of non-standard IVIG was relatively common in this cohort due to a primary misdiagnosis, particularly among patients from non-Cardiology departments. Therefore, pediatricians should improve the recognition and understanding of the diverse clinical manifestations of KD patients with neurological involvements, which may contribute to the prompt usage of standard IVIG therapy and in turn decrease the incidence of IVIG resistance in this populations. 


\section{Supplementary information}

Supplementary information accompanies this paper at https://doi.org/10. 1186/s12969-020-00452-7.

Additional file 1: Supplemental material 1. The comparison of clinical characteristic and laboratory results of patients with Kawasaki disease underwent CSF examination.

Additional file 2: Supplemental material 2. Comparison of clinical data between the groups of IVIG-response and IVIG-resistance in KD.

Additional file 3: Supplemental material 3. A multivariate logistic regression model for IVIG resistance in patients with $\mathrm{KD}$.

\section{Abbreviations}

KD: Kawasaki disease; KDSS: Kawasaki disease shock syndrome;

CALs: Coronary artery lesions; IVIG: Intravenous immunoglobulin; WCSUHSCU: West China Second University Hospital of Sichuan University; BSA: Body surface area; CSF: Cerebrospinal fluid; FLACC scale: Face, Legs, Activity, Cry, Consolability scale; RCA: Right coronary artery; LAD: Left anterior descending artery; LCX: Left circumflex coronary artery; LDH: Lactate dehydrogenase; WBC: White blood cell; CRP: C-reactive protein; Hb: Hemoglobin; Cr: Creatinine; TB: Total bilirubin; ALB: Albumin; MRI: Magnetic resonance imaging; MRA: Magnetic resonance angiography; SPECT: Single-photon emission computed tomography

\section{Acknowledgments}

Not Applicable.

\section{Authors' contributions}

$L X L$ drafted the manuscript, contributed to the data collection, interpreted the statistical analysis and approved the final manuscript as submitted. ZKY provided Table 1, contributed to the data collection, study design and as well as approved the final manuscript as submitted. HYM provided major treatment on these patients while admitted, contributed to the study design, approved financial support and as well as approved the final manuscript as submitted. WM provided Table 2, contributed to the data collection and approved the final manuscript as submitted. LL contributed to the data collection and approved the final manuscript as submitted. SSR contributed to the data collection and approved the final manuscript as submitted. WC conceived conception and designed the study, contributed to the data collection and approved the final manuscript as submitted.

\section{Funding}

This work was supported by National Natural Science Foundation of China (No. 81800288, No. 81741026 and No. 81571515), Science-technology Support Plan Projects in Sichuan province (No. 2019YFS0243, No. 2017SZ0117), Technology Innovation Research and Development Project of Chengdu Science and Technology Bureau (No 2018-YF05-00249-SN).

\section{Availability of data and materials}

All data generated or analyzed during this study are included in this published article and the supplementary files.

\section{Ethics approval and consent to participate}

Written informed consent was obtained from the parents following a full explanation of the nature of the study. The University Ethics Committee on Human Subjects at Sichuan University approved the study.

\section{Consent for publication}

Written consent obtained.

\section{Competing interests}

None of authors declared any conflict of interests.

\section{Author details}

'Department of Pediatric Cardiology, West China Second University Hospital, Sichuan University, No. 20, 3rd section, South Renmin Road, Chengdu 610041, Sichuan, China. ${ }^{2}$ Key Laboratory of Birth Defects and Related Diseases of Women and Children (Sichuan University), Ministry of Education, Chengdu, Sichuan, China. ${ }^{3}$ Key Laboratory of Development and Diseases of
Women and Children of Sichuan Province, West China Second University Hospital, Sichuan University, Chengdu, Sichuan, China. ${ }^{4}$ The Cardiac Development and Early Intervention Unit, West China Institute of Women and Children's Health, West China Second University Hospital, Sichuan University, Chengdu, Sichuan, China. ${ }^{5}$ West China Medical School of Sichuan University, Chengdu, Sichuan, China.

Received: 2 January 2020 Accepted: 3 July 2020

Published online: 14 July 2020

\section{References}

1. McCrindle BW, Rowley AH, Newburger JW, Burns JC, Bolger AF, Gewitz M, et al. Diagnosis, treatment, and long-term Management of Kawasaki Disease: a scientific statement for health professionals from the American Heart Association. Circulation. 2017;135(17):e927-99.

2. Fabi M, Corinaldesi E, Pierantoni L, Mazzoni E, Landini C, Bigucci B, et al. Gastrointestinal presentation of Kawasaki disease: A red flag for severe disease? PloS one. 2018:13(9):e0202658.

3. Singh S, Gupta A, Jindal A, Gupta A, Suri D, Rawat A, et al. Pulmonary presentation of Kawasaki disease-a diagnostic challenge. Pediatr Pulmonol. 2018;53(1):103-7.

4. Colomba C, La Placa S, Saporito L, Corsello G, Ciccia F, Medaglia A, et al. Intestinal involvement in kawasaki disease. J Pediatr. 2018;202:186-93.

5. Tizard EJ. Complications of Kawasaki disease. Curr Paediatr. 2005;15(1):62-8.

6. Wright H, Geddes J, Newburger JW, Burgner D. Facial nerve palsy complicating Kawasaki disease. \%A Wright H. Pediatrics. 2008;122(3):e783-5.

7. Takagi K, Umezawa T, Saji T, Morooka K, Matsuo N. Meningoencephalitis in Kawasaki disease. No To Hattatsu. 1990;22(5):429-35.

8. Husain E, Hoque E. Meningoencephalitis as a presentation of Kawasaki disease. J Child Neurol. 2006;21(12):1080-1.

9. Tabarki B, Mahdhaoui A, Selmi H, Yacoub M, Essoussi AS. Kawasaki disease with predominant central nervous system involvement. Pediatric Neurol. 2001:25(3):239-41.

10. Sundel RP, Newburger JW, Mcgill T, Cleveland SS, Miller WW, Berry B, et al. Sensorineural hearing loss associated with Kawasaki disease. Otolaryngol Online J. 2014;4(2):371-7

11. Rodriguez-Gonzalez M, Castellano-Martinez A, Perez-Reviriego AA. Atypical Presentation of Incomplete Kawasaki Disease: A Peripheral Facial Nerve Palsy. \%A Rodriguez-Gonzalez M. J Emerg Med. 2018:55(1):118-20.

12. Shiba T, Hamahata K, Yoshida A. Acute encephalopathy with biphasic seizures and late reduced diffusion in Kawasaki disease. Pediatr Int. 2017; 59(12):1276-8.

13. Dhawan SR, Vaidya PC, Suri D, Singh S, Singhi PD. Infantile Kawasaki disease presenting as acute meningoencephalitis. Int J Rheumatic Dis. 2017;20(12): 2225-6.

14. Stowe RC. Facial nerve palsy, Kawasaki disease, and coronary artery aneurysm. Eur J Paediatr Neurol. 2015;19(5):607-9.

15. Smith KA, Yunker WK. Kawasaki disease is associated with sensorineural hearing loss: a systematic review. Int J Pediatr Otorhinolaryngol. 2014;78(8): 1216-20.

16. Takanashi J, Shirai K, Sugawara Y, Okamoto Y, Obonai T, Terada H. Kawasaki disease complicated by mild encephalopathy with a reversible splenial lesion (MERS). J Neurol Sci. 2012;315(1-2):167-9.

17. Novo A, Pinto S, Prior AC, Alvares S, Soares T, Guedes M. Kawasaki disease and sensorineural hearing loss: an (un)expected complication. Eur J Pediatr. 2012;171(5):851-4.

18. Yoshikawa $\mathrm{H}$, Abe T. Febrile convulsion during the acute phase of Kawasaki disease. Pediatr Int. 2004;46(1):31-2.

19. Alves NR, Magalhaes CM, Almeida Rde F, Santos RC, Gandolfi L, Pratesi R. Prospective study of Kawasaki disease complications: review of 115 cases. Rev Assoc Med Bras (1992). 2011;57(3):295-300.

20. Sessler CN, Gosnell MS, Mary Jo G, Brophy GM, O'Neal PV, Keane KA, et al. The Richmond Agitation-Sedation Scale: validity and reliability in adult intensive care unit patients. Am J Respir Crit Care Med. 2002;166(10):1338-44.

21. Sessler CN, Grap MJ, Brophy GM. Multidisciplinary management of sedation and analgesia in critical care. Semin Respir Crit Care Med. 2001;22(02):211-26.

22. Merkel S, Voepellewis T, Shayevitz JR, Malviya S. The FLACC: a behavioral scale for scoring postoperative pain in young children. Pediatr Nurs. 1997; 23(3):293-7.

23. Lee Wong D, Morain BC. Wong and baker FACES pain rating scale; 1997. 
24. Kanegaye JT, Wilder MS, Molkara D, Frazer JR, Pancheri J, Tremoulet AH, et al. Recognition of a Kawasaki disease shock syndrome. Pediatrics. 2009; 123(5)::783-9.

25. Bayers S, Shulman ST, Paller AS. Kawasaki disease: Part II. Complications and treatment. J Am Acad Dermatol. 2013;69(4):513.e1-8.

26. Yeom JS, Cho JY, Woo HO. Understanding the importance of cerebrovascular involvement in Kawasaki disease. Korean J Pediatr. 2019; 62(9):334-9.

27. Korematsu S, Uchiyama S, Miyahara H, Nagakura T, Okazaki N, Kawano T, et al. The characterization of cerebrospinal fluid and serum cytokines in patients with Kawasaki disease. Pediatr Infect Dis J. 2007;26(8):750-3.

28. Oates-Whitehead RM, Baumer JH, Haines L, Love S, Maconochie IK, Gupta A, et al. Intravenous immunoglobulin for the treatment of Kawasaki disease in children. Cochrane Database Syst Rev. 2003;2003(4):CD004000. https:// pubmed.ncbi.nlm.nih.gov/14584002/.

29. Lo MS, Newburger JW. Role of intravenous immunoglobulin in the treatment of Kawasaki disease. Int J Rheumatic Dis. 2017:21(1):64-9.

30. Chen J, Ma B, Lin L, Xue Y. Treatment of Kawasaki disease by different doses of immunoglobulin: a meta analysis of efficacy and safety. Transl Pediatr. 2012;1(2):99-107.

31. Yeo J, Choi J. Effectiveness of medium-dose intravenous immunoglobulin (1 $\mathrm{g} / \mathrm{kg}$ ) in the treatment of kawasaki disease. Korean Circ J. 2010:40(2):81-5.

32. Uehara R, Belay ED, Maddox RA, Holman RC, Nakamura Y, Yashiro M, et al. Analysis of potential risk factors associated with nonresponse to initial intravenous immunoglobulin treatment among Kawasaki disease patients in Japan. Pediatr Infect Dis J. 2008;27(2):155.

\section{Publisher's Note}

Springer Nature remains neutral with regard to jurisdictional claims in published maps and institutional affiliations.

Ready to submit your research? Choose BMC and benefit from:

- fast, convenient online submission

- thorough peer review by experienced researchers in your field

- rapid publication on acceptance

- support for research data, including large and complex data types

- gold Open Access which fosters wider collaboration and increased citations

- maximum visibility for your research: over $100 \mathrm{M}$ website views per year

At $\mathrm{BMC}$, research is always in progress.

Learn more biomedcentral.com/submissions 